\title{
Risk Factors for Diabetic Ketoacidosis In Sohag University Hospitals
}

\author{
Mohamed Eid $^{\mathrm{a}^{*}}$, Imran Saber Mohammad ${ }^{\mathrm{a}}$, Adel Abdel-Aziz El-Sayed ${ }^{\mathrm{a}}$, Hamdy Saad \\ Mohamed ${ }^{\mathrm{a}}$.

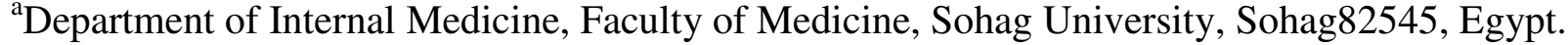

\begin{abstract}
Background: Diabetic ketoacidosis (DKA) is considered one of the pathological developments resulting from diabetes mellitus (DM), which threatens the patient's life. In Egypt, many factors participated in DKA prevalence, which increases the need for further deep studies.

Objectives: The current study aimed to measure and evaluate clinical profiles, the possible risk factors for DKA in Sohag Governorate, Egypt.

Patients and Methods: 60 patients diagnosed with DKA were recruited for six months between September 2018 and March 2019. The medical history and clinical investigations were applied to all participants. Data were collected and analyzed.

Results: The participants had a mean age of 32.76 \pm 18.8 years, and $62 \%$ were females. Most of the patients had a medical history of DM, and only $11.7 \%$ of them had a recent diagnosis. $55 \%$ of DKA patients had either respiratory tract or urinary tract infections $(26.6 \%$ and $16,6 \%$, respectively), where noncompliance for treatment was reported in $50 \%$ of cases. Lack of awareness about the DKA disease and its complication was reported in $45 \%$ of patients. Other comorbidities such as stroke (6.7\%), myocardial infarction (MI) (8.3\%), and acute pancreatitis (3.3\%) were reported, as well.

Conclusion: Many of the DKA patients from Sohag University Hospital, Sohag, Egypt, lack awareness about the disease and its risk factors. The major risk factors for DKA were either infection or less commitment to the medical regimens. More efforts should be paid to accommodate the health education weakness of diabetes and DKA risk factors.
\end{abstract}

Keywords: Diabetic ketoacidosis; risk factors; medical education.

\section{Introduction}

Diabetic ketoacidosis is one of the comorbidities which is commonly diagnosed among chronic DM patients and caused serious hyperglycemic symptoms (Onyiriuka and Ifebi, 2013). In the United States (US), the annual prevalence of DKA is $4.6-8$ for each 1000 DM type 1 patients (Savage et al., 2011). According to Kitabchi et al., (2009), the rate of hospitalization due to DKA, since the 1980s, is more than 100,000 patients per year, which costs more than a billion dollars of medical coverage for DM patients in the US. Clinical situations such as eating disorders and insulin omission, chronic diseases stress, or weight gain increase the risk of DKA by $20 \%$, particularly in young patients diagnosed with type 1 diabetes (Polonsky et al., 1994, Satti et al., 2013). According to the Indian study conducted by Poovazhagi (2014), DKA was suggested to increase the mortality rate of children in developing countries, such as India, Pakistan, and Middle Eastern countries, due to the higher

Copyright: (c) Eid et al. (2022) Immediate open access to its content on the principle that making research freely available to the public supports a greater global exchange of knowledge. Users have the right to Read, download, copy, distribute, print or share link to the full texts under a Creative Commons BY-NC-SA 4.0 International License. 
incidences of cerebral edema, sepsis, shock, and renal failures.

DKA is characterized by the discrepancy in the levels of insulin and other counter-regulatory hormones such as cortisol, glucagon, growth hormone, and catecholamine, which further results in serious metabolic derangements (Tan et al., 2012). DKA diagnosis involves the measurement of metabolic acidosis, hyperglycemia, and ketonemia (Chiasson et al., 2003). However, DKA might be equally diagnosed in both genders, males are subjected to its symptoms at earlier ages (Barski et al., 2011; Dabelea et al., 2014). The most common symptoms related to DKA are usually abdominal pain, dehydration, excessive urination, extreme thirst, nausea, vomiting, confusion, respiratory distress, and fruity breath (Ali, 2005).

One of the most characteristic features of DKA is that it can be diagnosed in all types of diabetes, particularly in patients who suffered from microbial infection or those classified as noncompliant to insulin treatment due to different psychological and socio-economic risk factors (Al-Rubeaan et al., 2011; Rahim et al., 2011; Tan et al., 2012). Besides, other diabetic comorbidities such as

\section{Patients and methods \\ Patients}

The current prospective observational study was conducted in the department of Internal medicine of Sohag University, Sohag, Egypt, between 1/9/2018 to 1/3/2019. The study included 60 diabetic patients at different diagnostic levels. The inclusion criteria included all hospitalized diabetic patients who were diagnosed with DKA according to the guidelines of the American Diabetic Association (ADA) (American Diabetes Association, 2019). All included stroke, acute MI, kidney failure, stroke, or acute pancreatitis participate in the development of DKA, as well (Rahim et al., 2011; Ahmed et al., 2014). The rapid diagnosis of DKA, level of severity, organ dysfunction, identification of risk factors, and other co-morbidities enable the initiation of adequate treatment which, consequently, affect the outcomes of the disease (Silverstein et al., 2005). So, this increases the necessity for appropriate management of the disease and its risk factors, which are thought to participate in the reduction of hospitalization and mortality rates (Hara et al., 2013). Besides, the implementation of appropriate educational programs about the risks of DKA and enabling of a sufficient supply of insulin and another antidiabetic medication should participate in reducing DKA incidence, as well (Hamed et al., 2017). Despite being one of the developing countries, few studies were conducted about DKA risk factors in Egypt (Bassyouni et al., 2012).

So, the current study aimed to estimate the risk factors associated with DKA among diabetic patients from Sohag University Hospital, Sohag, Egypt, to increase awareness and improve the outcomes.

patients had blood glucose $>250 \mathrm{mg} / \mathrm{dL}$, arterial $\mathrm{pH}$ of $\leq 7.30$, bicarbonate level of $\leq 18 \mathrm{mEq} / \mathrm{L}$, and Positive serum and urine ketones. Patients were classified according to the severity of diseases, where mild DKA with an arterial $\mathrm{pH}$ of 7.25-7.30, moderate DKA with an arterial $\mathrm{pH}$ of 7.0-7.24, and severe DKA with an arterial $\mathrm{pH}<7.0$ were considered (Kitabchi et al., 2009). On the other hand, other diabetic patients with either metabolic acidosis, acute and chronic renal failure, lactic acidosis and drugs, alcoholic ketosis, starvation, or 
hyperosmolar hyperglycemic nonketotic syndrome were excluded. Patients with ketonuria and high blood glucose, while the arterial $\mathrm{pH}$ is normal, were excluded, as well.

Patients were evaluated clinically where all necessary investigations such as blood glucose level, arterial blood gas analysis, levels of urinary ketones were performed for the diagnosis of DKA and its severity. Serum electrolytes were measured to assess hypo and hyperkalemia.

Informed consent was signed and collected from all patients. The study was approved by the Ethical Committee of Faculty of Medicine, Sohag University Hospital. It followed the guidelines of the declaration of Helsinki for medical research involving human subjects available from https://www.wma.net/policies-

post/wma-declaration-of-helsinki-ethicalprinciples-for-medical-research-involvinghuman-subjects/

\section{Assessment of risk factors}

The risk factors were assessed by investigating the medical history and clinical examination, according to the following criteria:

\section{Infection analysis}

The infection was estimated by stool analysis looking for Gastrointestinal (GIT) infection, urea, creatinine, urine complete examination looking for pus cell, chest XRay to confirm chest infection, the culture of appropriate samples to confirm infection, complete blood count looking for leukocytosis, and imaging, as guided by clinical presentation.

\section{Central nervous system (CVS)}

By CT scan of the brain.

3. Myocardial infarction (MI)

By electrocardiography, and estimation of the cardiac enzyme levels.

\section{Acute pancreatitis}

By measuring the levels of amylase, lipase, and abdominal CT scan.

\section{Socio-economic status}

A questionnaire-based assessment according to the updated and re-validated scoring system of Fahmy and El-Sherbini for measurement of socioeconomic status in health research in Egypt (El-Gilany et al., 2012). The scale included seven domains with a total score of 84 , where the higher score indicates better socio-economic status for education and culture, occupation, family possessions, economic status, home sanitation, and health care.

6. Awareness about the disease, its treatment, and complication

Patients were questioned about their knowledge about DKA disease, dietary adherence, commitment to insulin injection, and whether it was performed correctly. Besides, patients were asked about the disease-related factors such as DM duration, insulin or other treatment regimens, and possible causes of DKA such as missed insulin dose, illness-like infection, and the other factors. The readings of the last HbA1c (within three months), glucometer availability, and frequencies of home glucose and HbAlc checking were reported by patients as well.

\section{Compliance for treatment}

Patients were asked about regular intake of their treatment and whether the patient had stopped his treatment.

\section{Statistical analysis}

Data were analyzed by statistical package for social sciences (SPSS) version 12.0 and results were presented in tables.

\section{Results}

\section{Characteristics of the study population}

In the current study, 43 patients (72\%) were of type I DM, while 17 patients (28\%) typed II DM. Patients had mean ages of $32.76 \pm 18.8$, in which patients of type I DM had a mean age of $21.8 \pm 5.52$ years, and those of type II DM had a mean age of $59.9 \pm 9.9$ years. As shown in Table 1, 23 
patients (38\%) were males, and $37(62 \%)$

index

(BMI)

values

$(<25 \%)$.

were females. Patients had lower body mass

Table 1. Demographic data, clinical presentation and Investigation

\begin{tabular}{|c|c|c|c|c|}
\hline \multicolumn{2}{|c|}{ Variables } & Type I DM & Type II DM & Total \\
\hline \multicolumn{2}{|c|}{ Number of patients } & $43(72 \%)$ & $17(28 \%)$ & $60(100)$ \\
\hline \multicolumn{2}{|l|}{ Age (years)* } & $21.8 \pm 5.52(14-35)$ & $59.9 \pm 9.97(35-75)$ & $32.76 \pm 18.8(14-75)$ \\
\hline \multirow{2}{*}{ Gender } & Male & $14(32.6 \%)$ & $9(53 \%)$ & $23(38 \%)$ \\
\hline & female & $29(67.4 \%)$ & $8(47 \%)$ & $37(62 \%)$ \\
\hline \multicolumn{2}{|l|}{ BMI $\left(\mathbf{k g} / \mathbf{m}^{2}\right)^{\#}$} & $22.14 \pm 4.17$ & $27.35 \pm 2.76$ & $23.62 \pm 4.48$ \\
\hline \multicolumn{2}{|l|}{$\mathbf{p} \mathbf{H}^{\#}$} & $7.10 \pm 0.15$ & $7.18 \pm 0.05$ & $7.12 \pm 0.14$ \\
\hline \multirow{3}{*}{ Acidosis } & Mild & $8(18.6 \%)$ & $10(16.7 \%)$ & $2(11.8 \%)$ \\
\hline & Moderate & $26(60.5 \%)$ & $41(68.3 \%)$ & $15(88.2 \%)$ \\
\hline & severe & $9(20.9 \%)$ & $9(15 \%)$ & $0(0 \%)$ \\
\hline \multicolumn{2}{|c|}{ Abdominal pain } & $24(55.8 \%)$ & $3(17.6 \%)$ & $27(45 \%)$ \\
\hline \multicolumn{2}{|l|}{ Vomiting } & $30(69.8 \%)$ & $7(41.2 \%)$ & $37(61.7 \%)$ \\
\hline \multicolumn{2}{|c|}{$\begin{array}{l}\text { Disturbed Conscious } \\
\text { Level }\end{array}$} & $9(20.9 \%)$ & $4(23.5 \%)$ & $13(21.7 \%)$ \\
\hline \multicolumn{2}{|c|}{ Hypotension (mm Hg) } & $2(4.6 \%)$ & $3(17.6 \%)$ & $5(8.3 \%)$ \\
\hline \multicolumn{2}{|c|}{ Temperature $\left({ }^{\circ} \mathbf{C}\right)^{\#}$} & $37.2 \pm 0.44$ & $37.4 \pm 0.75$ & $37.2 \pm 0.55$ \\
\hline \multicolumn{2}{|c|}{ Blood sugar $(\mathrm{mg} / \mathrm{dl})^{\#}$} & $553 \pm 106$ & $530 \pm 62.3$ & $546.57 \pm 95.7$ \\
\hline \multirow{3}{*}{$\begin{array}{l}\text { Urine ketone } \\
\text { bodies }\end{array}$} & +2 & $11(18.3 \%)$ & $7(16.2 \%)$ & $4(23.5 \%)$ \\
\hline & +3 & $25(41.7 \%)$ & $19(44.1 \%)$ & $6(35.2 \%)$ \\
\hline & +4 & $24(40 \%)$ & $17(39.5 \%)$ & $7(41.1 \%)$ \\
\hline \multicolumn{2}{|c|}{$\mathbf{K}^{+}(\mathbf{m E q} / \mathbf{l})$} & $4.28 \pm 0.77$ & $4.20 \pm 0.79$ & $4.47 \pm 0.71$ \\
\hline
\end{tabular}




\begin{tabular}{|c|c|c|c|}
\hline Hgb $(\mathbf{g m} / \mathbf{d l})$ & $12.59 \pm 1.29$ & $12.9 \pm 1.17$ & $11.9 \pm 1.37$ \\
\hline WBC $(\mathbf{1 0} / \mathbf{u l})$ & $9.84 \pm 3.06$ & $9.02 \pm 2.63$ & $11.9 \pm 3.13$ \\
\hline Creatinine $(\mathbf{m g} / \mathbf{d l})$ & $1.05 \pm 0.43$ & $0.92 \pm 0.3$ & $1.34-0.57$ \\
\hline
\end{tabular}

* Mean \pm standard deviation (SD) (Min-Max) \#Mean \pm SD

\section{Risk factors}

In the current study, risk factors were assessed by a recent diagnosis of DM, existence of infection, co-morbidities, and awareness of diseases. As shown in Table 2, seven patients had been recently diagnosed with DM, of which six patients were type I and one patient was Type II. 33 patients suffered from infection equally distributed between type I and type II patients. The urinary tract infection (UTI), GIT, Respiratory tract, and skin infections were identified in $16.6 \%, 8.33 \%, 26.6 \%$, and $3.33 \%$ of the study population, respectively.

Table2. Risk factors for DKA patients.

\begin{tabular}{|c|c|c|c|}
\hline Variable & Type I (43) & Type II (17) & Total $(60)$ \\
\hline Newly diagnosed & $6(14 \%)$ & $1(5.9 \%)$ & $\begin{array}{c}7 \\
(11.7 \%) \\
\end{array}$ \\
\hline Infection & $24(55.8 \%)$ & $9(52.9 \%)$ & $\begin{array}{c}33 \\
(55 \%) \\
\end{array}$ \\
\hline 1. UTI & $7(16.2 \%)$ & $3(17.6 \%)$ & $10(16.6 \%)$ \\
\hline 2. GITinfection & $4(9.3 \%)$ & $1(5.8 \%)$ & $5 \quad(8.33 \%)$ \\
\hline 3. Respiratory tract infection & $12(27.9 \%)$ & $4(23.5 \%)$ & $16(26.6 \%)$ \\
\hline 4. Skin infection & $1(2.32 \%)$ & $1(5.8 \%)$ & $2(3.33 \%)$ \\
\hline Incompliance to treatment & $23(53.4 .4 \%)$ & $7(41.4 \%)$ & $30(50 \%)$ \\
\hline Low Socioeconomic status & $23(53.4 \%)$ & $3(17.6 \%)$ & $26(43 \%)$ \\
\hline Stroke & $0(0 \%)$ & $4(23.5 \%)$ & $4(6.7 \%)$ \\
\hline Myocardial infarction & $0(0 \%)$ & $5(29.4 \%)$ & $5(8.3 \%)$ \\
\hline Acute Pancreatitis & $1(2.3 \%)$ & $1(5.9 \%)$ & $2(3.3 \%)$ \\
\hline Awareness of the disease & $20(46.5 \%)$ & $7(41.1 \%)$ & $27(45 \%)$ \\
\hline
\end{tabular}


The results showed that half of the patients didn't commit to the prescribed medications, either because of the lowincome, less available insulin vials, the rawness of the appropriate injection performance, or simply because of the stress associated with a daily insulin injection. In accordance, $43 \%$ of patients had lower Socioeconomic status or lower income and health insurance, where most of them (23 patients) were type II.

The medical history of the participants showed that some DKA-related co-morbidities were represented, such as Stroke, MI, and Acute Pancreatitis. Most of these disorders were presented with type II patients as follows; four patients had a stroke, five patients had $\mathrm{MI}$, and one patient was diagnosed with Acute Pancreatitis. On the other hand, only one patient developed Acute Pancreatitis from the type I DM category.

The questionnaire involved many questions that reflect and measure the level of awareness of the DKA disease. The results showed a higher percentage of ignorance to the standard of knowledge about DKA and a poor level of health education among the study population, as shown in Table 2. A summary of the results is shown in Fig.1.

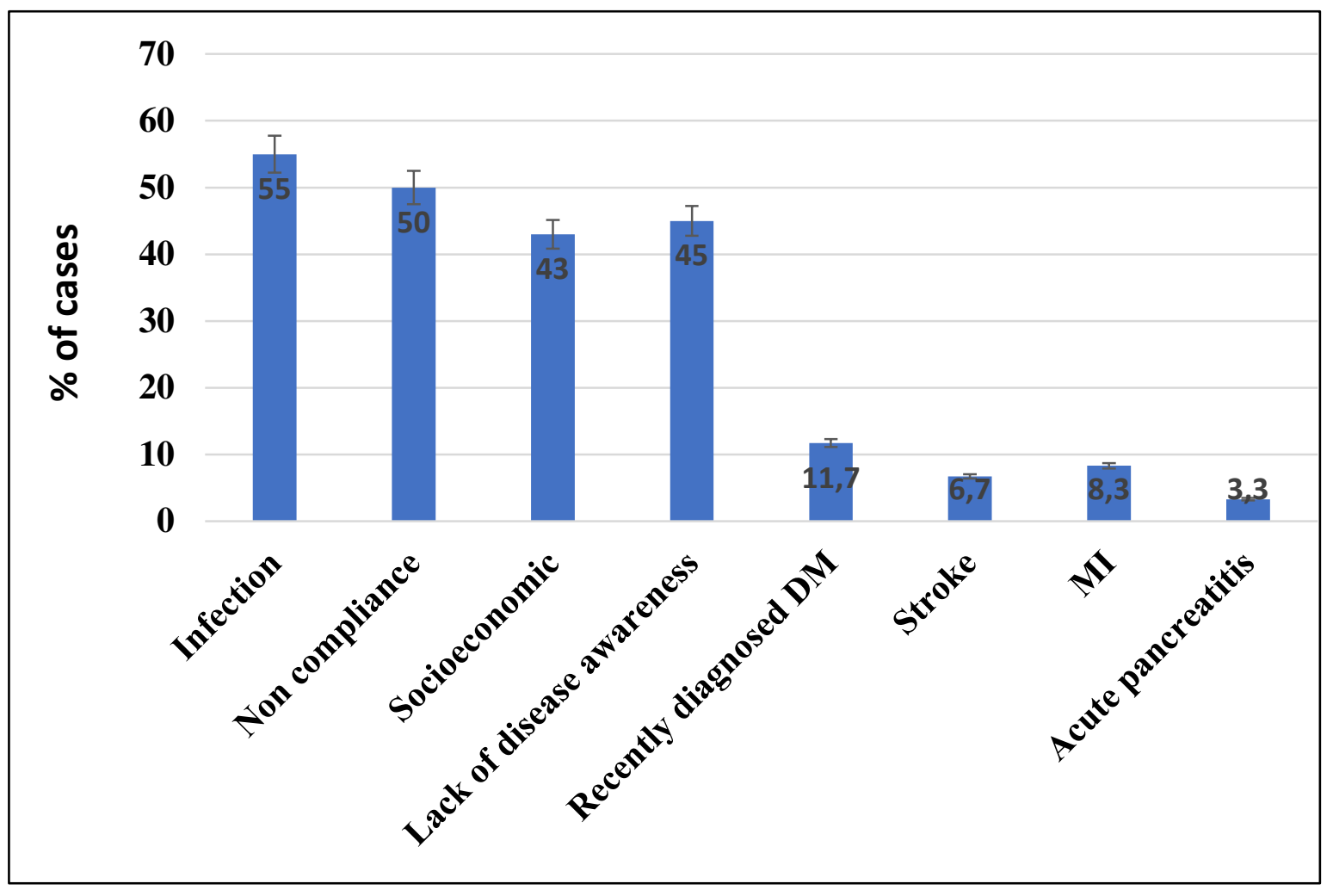

Fig. 1. Risk factors associated with DKA patients 


\section{Discussion}

Previous studies showed a higher incidence of DKA among patients of the two types of diabetes (Kitabchi et al., 2009; Savage et al., 2011; Onyiriuka and Ifebi, 2013). As shown before, DKA increases the annual hospitalization of DM patients up to 100,000 per year in the US, which increase gradually since 1980 (Kitabchi et al., 2009). The risk of DKA increased among young patients up to $30 \%$ because of the late diagnosis and other risk factors such as eating disorders and insulin resistance (Silverstein et al., 2005; Kitabchi et al., 2009).

In Egypt, the DKA situation is worse because of the large population, limitation in resources, and poor health coverage, which ranked Egypt as the ninth country in the incidence of DM (Type II) according to the International Diabetes Federation (IDF) guidelines (Hegazi et al., 2015). Another systemic review analyzed the data of 4,688 type I DM patients from the Arabian countries and reported that $46.7 \%$ were diagnosed with DKA, despite only $17 \%$ of DKA patients being Egyptians which reflects the low-diagnostic level (Zayed, 2016).

The objective of the current singlecenter study was to evaluate risk factors for DKA, which provides local statistics data about the precipitating factors of DKA. Several previous studies were conducted to evaluate this objective in different countries, worldwide. In the current study, the mean age of the studied population diagnosed with DKA was $32.76 \pm 18.8$, which included different age stages ranging from 14-75 years. In agreement, a previous study from Pakistan showed that DKA was detected in DM patients of 40 years (Rana et al., 2016), while another study from Tanta University, Egypt, showed that DKA was diagnosed at a lower mean age of 22 years (Hamed et al., 2017).
In the current study, most of the DKA patients were female (62\%). In contrast to our findings, previous studies showed that DKA was most prevalent among males by $\sim 58 \%$ (Newton and Raskin, 2004; Hamed et al., 2017) and 65.7\% (Rana et al., 2016).

Our findings showed that DM was recently diagnosed in seven patients $(11.7 \%)$ which is following the results of Rana et al., (2016) and showed that $10 \%$ of the DKA cases had a late diagnosis of DM, while another study showed a higher percentage of $23 \%$ of cases of the African Americans had recent DM profiles (Seyoum and Berhanu, 2007).

The reported symptoms in the current study showed that vomiting and abdominal pain were the most common in $61.7 \%$ and $45 \%$ of patients, respectively. In an agreement, a previous study reported vomiting and abdominal pain in $76 \%$ and $52 \%$ of cases, respectively (Rana et al., 2016). The consciousness level was altered in $21.7 \%$ of patients in our study, which is different from other previous studies of Rana et al., (2016) with a higher percentage $(68 \%)$ and lower percentage $(10 \%)$ in the study conducted by Miglani et al., (2000).

In the current study, the mean serum blood sugar level was $546.57 \pm 95.7 \mathrm{mg} / \mathrm{dL}$ (compared to the normal values at $\leq 140$ $\mathrm{mg} / \mathrm{dL}$ ) which indicated uncontrolled glycemia in the studied patients. In agreement with our findings, a previous study reported a higher mean blood sugar level of $(610.2 \mathrm{mg} / \mathrm{dl})$ (Yousaf and Chaudhry, 1997). The mean $\mathrm{pH}$ readings were neutral by $7.12 \pm 0.14$ which is following the findings of Yousaf and Chaudhry (1997), as well.

Interestingly, Infection was reported as the highest risk factor that was represented by $55 \%$ of the DKA cases. Furthermore, 12 patients of type I DM $(27.9 \%)$ and 4 patients of type II DM 
(23.5\%) had respiratory tract infections, while UTI was diagnosed in seven patients of Type I DM (16.2\%) and three patients of type II DM (17.6\%). In an agreement with our findings, a previous study from Tanta University Hospital, Egypt, it was reported that the main risk factor of DKA was an infection in $46.5 \%$ of the studied cases, where UTI was diagnosed in most cases $(31.2 \%)$, followed by the respiratory tract and GIT infections in $26.8 \%$ and $13.9 \%$ of cases, respectively (Hamed et al., 2017). Also, in the study of Hamed et al., (2017), other infections such as diabetic foot, ENT, skin, subcutaneous tissue, and mixed infections were diagnosed. Another study reported infection as the second most risk factor of DKA (Kitabchi et al., 2009). Another study suggested that DKA representation was reasoned for noncompliance to treatment in $45.5 \%$ of DM patients, late diagnosis of DM type $\mathrm{I}$ in $20.9 \%$, and infection in $27.2 \%$ (Bassyouni et al., 2012). In the latter study, the reported infections were either respiratory tract infection, UTI, gastroenteritis, diabetic foot, or abscess in most cases (Bassyouni et al., 2012). Similarly, multiple studies suggested different infections as the main cause of DKA (Newton and Raskin, 2004; Mbugua et al., 2005; Rana et al., 2016; Hamed et al., 2017).

In the current study, the poor commitment to treatment, particularly insulin, was represented in $50 \%$ of the studied population. Similar observations were reported in Harris et al., (2009) that reported in-compliance to insulin treatment in $59 \%$ of patients, whereas Kitabchi et al., (2009), reported it in $47 \%$ of the studied cases.

Finally, the levels of knowledge standards about the diseases were very poor and were reported in $45 \%$ of cases. This might be due to the poor educational level of the studied population, besides the non- availability of informative health education. Furthermore, some patients claimed that some physicians and health care providers didn't offer sufficient or informative instructions and guidelines about the disease, medications, and follow-up visits. These claims were common in the studies from other countries such as in the Pakistani cities Quetta (Ali et al., 1998) and Karachi (Afzal et al., 2005). Another Indian-guided survey-based study showed that $43 \%$ of the less-educated young DM patients were advised by some quacks and clergymen to stop the treatments prescribed by physicians and to use their uncertain prescriptions of folk medications (Miglani et al., 2000).

Similarly, another study reported that health education about DKA is tended to be ignored or neglected by patients, the general public, or even healthcare professionals (Umpierrez and Kitabchi, 2003). Furthermore, irregular follow-up was reported as one of the possible risk factors of DM complications, including DKA (Jacobson et al., 1997). Another study from Pakistan emphasized the importance of health education and the necessity of developed DM clinics at the society level (Chaudry et al., 2020).

In the current study, the low socioeconomic status was an important risk factor of DKA where $43 \%$ of the studied population had lower incomes. Similarly, in the study conducted by Al-Obaidi et al., (2019), the effect of the Socioeconomic Status among DM type I patients from Basrah, Iraq, suggested that many socioeconomic factors played key roles in DKA development.

MI is one of the comorbidities associated with DKA patients in the current study. As shown before $8.3 \%$ of DKA patients were diagnosed with MI. A similar study conducted in the National Institute of Diabetes and Endocrinology (NIDE), Egypt, 
revealed that MI was diagnosed in $6.4 \%$ of cases (Bassyouni et al., 2012).

As been reported before, the evaluation of DKA might be controlled by several socioeconomic and clinical factors such as appropriate health care coverage, proper health education, and feasible communications between patients and health care providers (Fasanmade et al., 2008).

\section{Conclusion}

The current study reported the possible risk factors associated with DKA in patients from Sohag, Egypt. Variable infections and poor commitment to insulin treatment are the most reported factors for DKA. Furthermore, the poor understanding and knowledge about DKA, besides, the low level of health education participated deeply in the development of DKA. The current findings suggested that training and educating the DKA patients about the disease and increasing the health costs coverage might decrease the severity level of DKA among Sohag patients. More studies are required to investigate more risk factors and diagnostic levels of DKA among diabetic patients.

\section{List of abbreviations}

ADA: American Diabetic Association; BMI: Body Mass Index; CVS: Central Nervous System; DKA: Diabetic ketoacidosis; DM: Diabetes Mellitus; GIT: Gastrointestinal; MI: Myocardial infarction; NIDE: National Institute of Diabetes and Endocrinology; US: United States; UTI: urinary tract infection; SD: Standard Deviation; SPSS: Statistical Package for Social Sciences.

\section{References}

Afzal A, Zaheer H, Jamalvi WA, Nisar K, Mazahir I (2005). Presentation and precipitating factors of Diabetic Ketoacidosis in children. Annals of Abbasi Shaheed Hospital \& Karachi Medical college.10: 766-774.
Ahmed AU, Rahim MA, Rahman MR, Nazim RF, Uddin KN (2014). Diabetic Ketoacidosis: Pattern of Precipitating Causes. Journal of Enam Medical College. 4(2): 94-97.

Ali A, Hassan Z, Wasim Ahmed J, Kashif N, Iqbal M (2005). Presentation and precipitating factors of diabetic Ketoacidosis in children. Annals Abbassi Shaheed Hospital and Karachi Medical and Dental College. 10 (2): 766-774.

Ali M, Khalid GH, Pirkani GS (1998). Level of health education in patients with type II diabetes mellitus in Quetta. Journal Of Pakistan Medical Association: JPMA. 48(11):334-336.

Al-Obaidi AH, Alidrisi HA, Mansour AA (2019). Precipitating Factors for Diabetic Ketoacidosis among Patients with Type 1 Diabetes Mellitus: The Effect of Socioeconomic Status. International Journal of Diabetes and Metabolism.25:52-60.

Al-Rubeaan KA, Aftab SA, Alotaibi MS, Alghamdi AA, Rafiullah MR (2011). Clinico-laboratory characteristics of diabetic keto acidosis in adults in a tertiary hospital in Saudi Arabia. European Review for Medical and Pharmacological Sciences. 15(10):1202-1206.

American Diabetes Association, 2019. 5. Lifestyle Management: Standards of Medical Care. In: Diabetes-2019. Diabetes Care, pp: S46-S60.

Barski L, Harman-Boehm I, Nevzorov R, Rabaev E, Zektser M, Jotkowitz AB, et al (2011). Gender-related differences in clinical characteristics and outcomes in patients with diabetic ketoacidosis. Gender Medicine, 8(6):372-377.

Bassyouni A, El Ebrashy I, El Hefnawy H (2012). Epidemiology of diabetic ketoacidosis in National Institute of 
Diabetes and Endocrinology. Endocrine Abstracts. 29:659.

Chaudry NT, Farid R, Khan SJ, Imtiaz Y (2020). Diabetes Mellitus: The Association of Clinical Pattern and Complications with Type of Glycaemic Control. Annals of King Edward Medical University, 5(1):4-7.

Chiasson JL, Aris-Jilwan N, Bélanger R, Bertrand S, Beauregard H, Ékoé JM, et al (2003). Diagnosis and treatment of diabetic ketoacidosis and the hyperglycemic hyperosmolar state [published correction appears in CMAJ. 2003 May 13;168(10):1241].Canadian Medical Association Journal, 168(7):859-866.

Dabelea D, Rewers A, Stafford JM, Standiford DA, Lawrence JM, Saydah S, et al (2014). Trends in the prevalence of ketoacidosis at diabetes diagnosis: the SEARCH for diabetes in youth study. Pediatrics, 133(4):e938-e945.

El-Gilany A, El-Wehady A, El-Wasify M (2012). Updating and validation of the socioeconomic status scale for health research in Egypt. Eastern Mediterranean Health Journal, 18(9): 962968.

Fasanmade OA, Odeniyi IA, Ogbera AO (2008). Diabetic ketoacidosis: diagnosis and management. African Journal of Medicine and Medical Sciences, 37(2):99-105.

Hamed ZS, Gawaly AM, Abbas KM, El Ahwal LM (2017). Epidemiology of infection as a precipitating factor for diabetic ketoacidosis at Tanta University Hospital. Tanta Medical Journal, 45:68-72

Hara JS, Rahbar AJ, Jeffres MN, Izuora KE (2013). Impact of a hyperglycemic crises protocol. Endocrine practice,19(6):953-62.
Harris MA, Freeman KA, Beers M (2009). Family therapy for adolescents with poorly controlled diabetes: initial test of clinical significance. Journal of pediatric psychology, 34(10):1097-1107.

Hegazi R, El-Gamal M, Abdel-Hady N, Hamdy $\mathbf{O}$ (2015). Epidemiology of and Risk Factors for Type 2 Diabetes in Egypt. Annals of Global Health, 81(6):814-820.

Jacobson AM, Hauser ST, Willett J, Wolfsdorf JI, Herman L (1997). Consequences of irregular versus continuous medical follow-up in children and adolescents with insulin-dependent diabetes mellitus. The Journal of pediatrics, 131(5):727-733.

Kitabchi AE, Umpierrez GE, Miles JM, Fisher JN (2009). Hyperglycemic crises in adult patients with diabetes. Diabetes Care, 32(7):1335-1343.

Kitabchi AE, Umpierrez GE, Murphy MB, Barrett EJ, Kreisberg RA, Malone JI, et al (2001). Management of hyperglycemic crises in patients with diabetes. Diabetes Care, 24(1):131-153.

Mbugua PK, Otieno CF, Kayima JK, Amayo AA, McLigeyo SO (2005). Diabetic ketoacidosis: clinical presentation and precipitating factors at Kenyatta National Hospital, Nairobi. East African Medical Journal, 82(12 Suppl):S191-S196.

Miglani S, Sood A, Shah P (2000). Selfreported attitude and behavior of young diabetics about discussing their disease. Diabetes research and clinical practice, 48(1):9-13.

Miglani S, Sood A, Shah P (2000). Selfreported attitude and behavior of young diabetics about discussing their disease. Diabetes Research and Clinical Practice, 48(1):9-13. 
Newton CA, Raskin P (2004). Diabetic ketoacidosis in type 1 and type 2 diabetes mellitus: clinical and biochemical differences. Archives of internal medicine, 164(17):1925-1931.

Onyiriuka AN, Ifebi E (2013). Ketoacidosis at diagnosis of type 1 diabetes in children and adolescents: frequency and clinical characteristics. Journal of diabetes and metabolic disorders, 12(1):47.

Polonsky WH, Anderson BJ, Lohrer PA, Aponte JE, Jacobson AM, Cole CF (1994). Insulin omission in women with IDDM. Diabetes Care, 17(10):1178-1185.

Poovazhagi V (2014). Risk factors for mortality in children with diabetic keto acidosis from developing countries. World Journal of Diabetes, 5(6):932-938.

Rahim M, Uddin K, Zaman S, Musa A, Rahman M, Hossain M, et al (2011). Clinical spectrum and management of diabetic ketoacidosis: experience in a tertiary care hospital. BIRDEM Medical Journal, 1(1):15-20.

Rana SA, Malik WA, Babar MA (2016). To Evaluate Various Precipitating Factors for Diabetic Ketoacidosis. Pakistan Journal of Medical \& Health Sciences, 10(2):482485.

Satti SA, Saadeldin IY, Dammas AS (2013). Diabetic Ketoacidosis in children admitted to Pediatric Intensive Care Unit of King Fahad Hospital, Al-Baha, Saudi Arabia: Precipitating factors, epidemiological parameters and clinical presentation. Sudanese Journal of Paediatrics, 13(2):24-30.

Savage MW, Dhatariya KK, Kilvert A, Rayman G, Rees JA, Courtney CH, et al (2011). Joint British Diabetes Societies guideline for the management of diabetic ketoacidosis. Diabetic Medicine, 28(5):508515.

Seyoum B, Berhanu P(2007). Profile of diabetic ketoacidosis in a predominantly African American urban patient population. Ethnicity and disease, 17(2):234.

Silverstein J, Klingensmith G, Copeland K, Plotnick L, Kaufman F, Laffel L, et al (2005). Care of children and adolescents with type 1 diabetes: a statement of the American Diabetes Association. Diabetes care, 28(1):186-212.

Tan H, Zhou Y, Yu Y (2012). Characteristics of diabetic ketoacidosis in Chinese adults and adolescents-a teaching hospital-based analysis. Diabetes research and clinical practice, 97(2):306-312.

Umpierrez GE, Kitabchi AE (2003). Diabetic ketoacidosis: risk factors and management strategies. Treatments in Endocrinology, 2(2):95-108.

Yousaf M, Chaudhry S(1997). Clinical and laboratory profile of episodes of diabetic ketoacidosis in Madina Al-Munawarah, Saudi Arabia Professional Medical JournalQuarterly, 4(2): 162-165.

Zayed H (2016). Epidemiology of diabetic ketoacidosis in Arab patients with type 1 diabetes: a systematic review. International Journal of Clinical Practice, 70(3):186-195. 